\title{
Engineering and Harnessing Giant Atoms in High-Dimensional Baths: A Proposal for Implementation with Cold Atoms
}

\author{
A. González-Tudela, ${ }^{1, *}$ C. Sánchez Muñoz, ${ }^{2}$ and J. I. Cirac ${ }^{3}$ \\ ${ }^{1}$ Instituto de Física Fundamental IFF-CSIC, Calle Serrano 113b, Madrid 28006, Spain \\ ${ }^{2}$ Clarendon Laboratory, University of Oxford, Oxford OX13PU, United Kingdom \\ ${ }^{3}$ Max-Planck-Institut für Quantenoptik Hans-Kopfermann-Str. 1. 85748 Garching, Germany
}

(Received 4 January 2019; published 21 May 2019)

\begin{abstract}
Emitters coupled simultaneously to distant positions of a photonic bath, the so-called giant atoms, represent a new paradigm in quantum optics. When coupled to one-dimensional baths, as recently implemented with transmission lines or SAW waveguides, they lead to striking effects such as chiral emission or decoherence-free atomic interactions. Here, we show how to create giant atoms in dynamical state-dependent optical lattices, which offers the possibility of coupling them to structured baths in arbitrary dimensions. This opens up new avenues to a variety of phenomena and opportunities for quantum simulation. In particular, we show how to engineer unconventional radiation patterns, like multidirectional chiral emission, as well as collective interactions that can be used to simulate nonequilibrium many-body dynamics with no analog in other setups. Additionally, the recipes we provide to harness giant atoms in high dimensions can be exported to other platforms where such nonlocal couplings can be engineered.
\end{abstract}

DOI: 10.1103/PhysRevLett.122.203603

The design and exploration of novel forms of light-matter interaction have been a driving force in quantum optics triggering both fundamental and technological advances. A paradigmatic example of this was the observation that atomic lifetimes renormalize within cavities [1], which opened the field of cavity QED $[2,3]$. This seemingly simple light-matter coupling led to many other fundamental discoveries, such as the creation of mixed light-matter particles (polaritons), and applications, e.g., in quantum information [4]. Another timely example is the interaction of (natural or artificial) emitters with the structured propagating photons (or matter waves) which appear in nanophotonic structures [5-14], circuits [15-17], or state-dependent optical lattices [18-22]. In these systems, the bath displays structured energy dispersions, leading to a plethora of effects absent in other environments. On the fundamental level, they generate nonexponential relaxations [23-29], whereas in the more applied perspective they lead to the emergence of bound states outside [30-35] or in the continuum [36-45], which can be harnessed for (out-of) equilibrium quantum simulation [46-49].

In all these setups the emitters are typically much smaller than their associated wavelength, leading to inherently local light-matter couplings. This picture, however, has been recently challenged with the design of the so-called "giant atoms," which are emitters coupled to several points of SAW waveguides [50-54] or transmission lines [55] separated beyond their characteristic wavelength. These giant atoms represent another paradigm change in quantum optics since the coupling to different bath positions induces strong interference effects which can be exploited for applications [20,21,56-58]. For instance, when coupled to one-dimensional baths they lead to decoherence-free atomic interactions [58], or to chiral emission [20,21] without exploiting polarization, something impossible to realize with "small" emitters. Exporting this paradigm to higher dimensional baths, where, for example, quantum simulation will show its full power, is a desirable, but challenging, goal. On the one hand, to our knowledge there is still no implementation to do so, since wiring up high-dimensional circuits becomes complicated. On the other hand, even if achieved, it is not obvious how to harness giant atoms when coupled to high-dimensional baths. The reason is that the resonant photons mediating the interactions, defined by the isofrequencies of $\omega(\mathbf{k})$ at the emitters' frequency, are contours (or surfaces) in $\mathbf{k}$ space, instead of points, making perfect interference more difficult.

In this Letter, we address both issues showing the following: (i) A proposal to engineer effective giant-atom coupling to baths with high dimensions. We use ultracold atoms in dynamical state-dependent optical lattices $[18,19,22]$ (see Fig. 1), such that by moving the relative position between the potentials [59-61] fast enough, the giant emitter couples effectively to several bath positions. (ii) A way to harness them to observe phenomena with no analog in other setups by coupling them to structured photonic reservoirs with a van Hove singularity [37-39,49]. In particular, we show how giant quantum emitters (QEs) can modify the non-Markovian nature of the dynamics, and lead to unconventional emission patterns, e.g., chiral emission in one or several directions, which translate into unconventional collective $\mathrm{QE}$ interactions when several 




FIG. 1. (a) State-dependent optical lattice scheme to simulate quantum optical phenomena: one deep lattice $V_{b}\left(\mathbf{R}_{b}\right)$ (blue) traps the atomic state that mimics the QE behavior, whereas a shallower one, $V_{a}\left(\mathbf{R}_{a}\right)$, lets matter-wave propagation at rate $J$. The two atomic states can be connected through a local laser(s) or microwave field (green) with strength $\Omega_{\mathbf{n}_{j}}$. The relative position between the lattices, and of the local laser can be dynamically tuned $\mathbf{R}_{b}(t)$. (b) Pictorial representation on how the effective giant atom couplings emerge from the stroboscopic movement between the lattices.

of them couple to the bath. Even though we make the discussion of items (i) and (ii) together throughout this Letter, the recipes that we provide for (ii) can be exported to other implementations where such couplings can be engineered.

Let us first recall how to obtain the standard quantum optical Hamiltonian with ultracold atoms [18,19,22]; see Fig. 1(a). One needs an atom with two states $a$ and $b$ subject to different potentials $V_{a, b}(\mathbf{R})$, whose dimensionality can be optically controlled [62]. The $b$ atoms are trapped in a deep potential such that they mostly localize within a lattice site, and in the strongly interacting regime, which means that there will be at most one $b$ excitation per lattice site such that their excitations can be represented by spin operators $\sigma_{g e}^{\mathbf{n}_{j}}$, with $\sigma_{\alpha \beta}^{\mathbf{n}}=|\alpha\rangle_{\mathbf{n}}\langle\beta|$. On the contrary, when the atoms are in the $a$ state, they can hop to their nearest neighbours at a rate $J$ without interactions, mimicking photon propagation. In addition, one needs an extra field that transfers the $b$ excitations into $a$ ones (and vice versa), which can be obtained via a Raman or microwave transition $[22,63]$ (or a direct one in the case of alkalineearth atoms [64-66]). Let us denote as $\Omega_{\mathbf{n}_{j}}$ the $a-b$ coupling at site $\mathbf{n}_{j}$, which can be controlled in both magnitude and phase though the lasers. As derived in Refs. [18,19], the Hamiltonian describing the dynamics of the excitations of the $a$ and $b$ atoms mimics the one standard light-matter interactions, that is, $H=H_{S}+H_{B}+H_{\text {int }}$, where

$$
\begin{gathered}
H_{S}=\omega_{e} \sigma_{e e}, \quad H_{B}=\sum_{\mathbf{k}} \omega(\mathbf{k}) a_{\mathbf{k}}^{\dagger} a_{\mathbf{k}}, \\
H_{\mathrm{int}}=\left(\Omega_{\mathbf{n}_{e}} a_{\mathbf{n}_{e}}^{\dagger} \sigma_{g e}+\text { H.c. }\right),
\end{gathered}
$$

where for illustration we restrict to a single QE, dropping the superindex in $\sigma_{\alpha, \beta}$. The $a_{\mathbf{k}}\left(a_{\mathbf{k}}^{\dagger}\right)$ are the annihilation (creation) operator of a matter-wave excitation with momentum $\mathbf{k}$, whose energy dispersion $\omega(\mathbf{k})$ is controlled by the geometry of $V_{a}(\mathbf{R})$. The $\mathrm{QE}$ is in the strong confinement limit such that its coupling will be local like with optical photons [67].

To effectively transform this local coupling into a nonlocal one among $\left\{\mathbf{n}_{\alpha}\right\}_{\alpha=1}^{N_{p}}$ positions, one can dynamically move the relative position between the $V_{a, b}(\mathbf{R})$ potentials in a periodic fashion, e.g., changing the relative phase between the lasers creating the potentials [62]. If the movement is adiabatic, that is $|\dot{\mathbf{R}}(t)| \ll d \omega_{t}$ for all $t$, where $d$ is the ground state size, and $\omega_{t}$ the trap frequency [59-61], the atoms remain in their motional ground state and can still be described by a Hamiltonian as in Eqs. (1) and (2) but with time-dependent parameters. For example, assuming that the simulated QE probes the $\left\{\mathbf{n}_{\alpha}\right\}_{\alpha=1}^{N_{p}}$ positions and that the laser parameters change as needed in each position, the Hamiltonian will now read

$$
H_{\mathrm{int}} \rightarrow H_{\mathrm{int}, \operatorname{mov}}(t)=\sum_{\alpha=1}^{N_{p}}\left[\Omega_{\mathbf{n}_{\alpha}}(t) a_{\mathbf{n}_{\alpha}}^{\dagger} \sigma_{g e}+\text { H.c. }\right] .
$$

Now, to formally derive how the desired nonlocal couplings emerge using Floquet analysis, we consider that the QE moves periodically along $N_{p}$ positions with period $T$ (and frequency $\omega=2 \pi / T$ ), probing each position during a constant time interval $T / N_{p}$ with coupling strength $g_{\mathbf{n}_{\alpha}}$ [68]. With that assumption, we can apply Floquet theory [69] to obtain an effective Hamiltonian description in the high-frequency limit. To the lowest order, it corresponds to the nonlocal light-matter couplings that we want to obtain (see Supplemental Material [70]):

$$
H_{\text {inteff }} \approx \sum_{\alpha=1}^{N_{p}}\left(\frac{g_{\mathbf{n}_{\alpha}}}{N_{p}} a_{\mathbf{n}_{\alpha}}^{\dagger} \sigma_{g e}+\text { H.c. }\right),
$$

where $g_{\mathbf{n}_{\alpha}} / N_{p}$ is the time average of $\Omega_{\mathbf{n}_{\alpha}}(t)$. We can also calculate the next-order term contribution which is of order $\sim 4\left|g_{\max }\right|^{2} N_{p}^{2} \xi[3] /\left(\pi^{2} \omega\right) \ll \max \left|g_{\mathbf{n}_{\alpha}}\right|$ for our situations of interest. Summing up, to obtain the desired behavior the periodic movement has to be slow enough to stay within the lowest band of the tight-binding Hamiltonians of Eqs. (1) and (2), but fast compared to the induced QE timescales, such that it effectively couples to several positions, i.e., $\omega_{t} \gg \omega(L / d) \gg \max \left|g_{\mathbf{n}_{\alpha}}\right|$ (assuming a constant speed over the distance $L$ that we displace the potentials). Since the couplings are tunable and they can always be made small, the lower bound of these inequalities will be ultimately provided by the decoherence rate $\Gamma^{*}$ of the setup, which should be smaller than the simulated parameters. To provide some estimation, we can first take the recent realization of our proposed setup [22], where two hyperfine ${ }^{87} \mathrm{Rb}$ levels were used to engineer the optical potentials, $|a, b\rangle=\left|F=1 / 2, m_{F}=-1 / 0\right\rangle$, with trap depths of the order $\omega_{t} \sim 2 \pi \times 10 \mathrm{kHz}$, and typical decoherence rates 


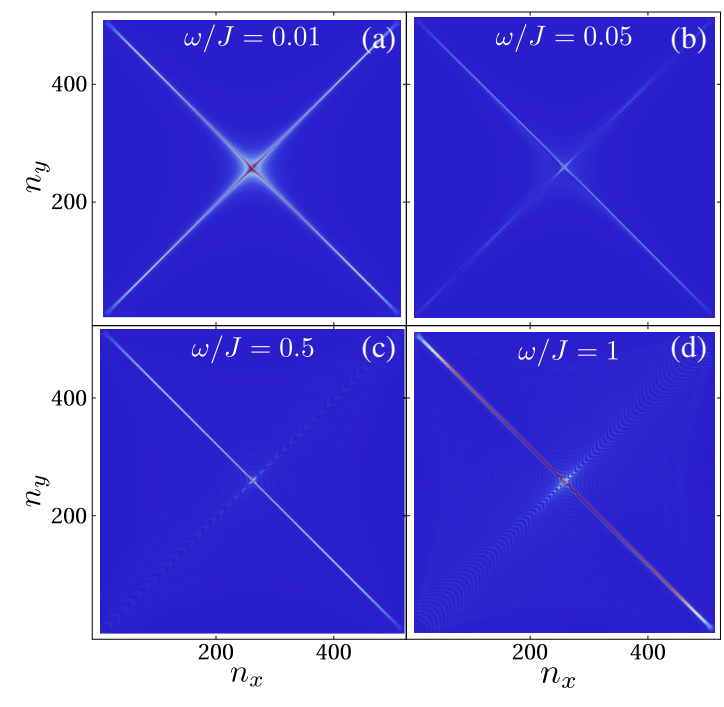

FIG. 2. Bath population at a time $t J=N / 4$ after the deexcitation of a single QE that moves between two lattice sites at positions $(0,0),(1,1)$, such that $g_{\mathbf{n}_{1}\left[\mathbf{n}_{2}\right]}=g \cos ^{2}(\omega t / 2)\left[\sin ^{2}(\omega t / 2)\right]$, with $g=0.1 J$ and $\omega$ as depicted in the legend. Bath linear size is $N=512$.

$\sim 10-100 \mathrm{~Hz}$. Another possibility is to use the ground or excited metastable state in alkaline-earth atoms (see Ref. [64] for a concrete proposal with strontium). This platform shows similar $\omega_{t}$, but decoherence can be substantially decreased since it will be mostly determined by the excited state lifetime which can be $\Gamma^{*} /(2 \pi) \lesssim 0.01 \mathrm{~Hz}$, thus leaving several orders of magnitude to adiabatically move the lattice.

Let us now show how to exploit giant QEs coupled to higher dimensional baths to obtain phenomena with no analog in other setups. In particular, we illustrate it by studying the spontaneous decay of an excited QE coupled to a two-dimensional bath with $\omega(\mathbf{k})=\omega_{a}-$ $2 J\left[\cos \left(k_{x}\right)+\cos \left(k_{y}\right)\right]$. When the QE interacts locally in space with frequency $\omega_{e}=\omega_{a}$, it couples equally to all the resonant k's defined by $k_{x} \pm k_{y}= \pm(\mp) \pi$. This contour, which includes points with zero group velocity $\left[\mathbf{v}_{g}(0, \pm \pi)=\mathbf{v}_{g}( \pm \pi, 0)=(0,0)\right]$ responsible for a van Hove singularity in the density of states [71], leads to two remarkable effects in the QE spontaneous decay [37-39]: (i) its emission pattern is highly anisotropic, as shown in Fig. 2(a), emitting mostly in four directions with some diffraction due to the inhomogeneous group velocity of the wave packet; (ii) its dynamics is intrinsically nonMarkovian due to divergence of the density of states at this frequency $[38,39]$. Now, we will show how building upon this behavior, giant QEs can lead to very flexible and unusual emission patterns and interactions.

Quasi-1D emission.-First, we show how to cancel the emission in one of the diagonals of Fig. 2(a) by coupling to two lattice sites $\mathbf{n}_{1 / 2}=(0,0) /(1,1)$. To numerically show how the Floquet averaged Hamiltonian $H_{\text {int,eff }}$ emerges, we assume that the movement between the lattices is such that $\Omega_{\mathbf{n}_{1}}(t)=g \cos ^{2}(\omega t / 2), \Omega_{\mathbf{n}_{2}}(t)=g \sin ^{2}(\omega t / 2)$, and solve the dynamics using $H_{\text {int,mov }}(t)$. In Figs. 2(b)-2(d) we plot the bath population in real space after a time $t J=N / 4$ using $g=0.1 \mathrm{~J}$, and for several $\omega$ 's. As expected, for $\omega \ll g$, the emission occurs in four directions as if the QE was locally coupled. However, as $\omega$ increases, the interference between the bath emission in two different points occurs, until it cancels the emission in one of the diagonals. This behavior can be understood from the asymptotic bath state in the perturbative limit [39]:

$$
C_{\mathbf{k}}(t \rightarrow \infty) \propto \frac{G(\mathbf{k}) e^{-i \omega(\mathbf{k}) t}}{\omega(\mathbf{k})-\omega_{e}+i \Gamma_{M} / 2},
$$

where $\Gamma_{M}$ is the Markovian decay rate, and $G(\mathbf{k})$ is the effective light-matter coupling between the emitter and the $\mathbf{k}$ modes, $H_{\text {int,eff }}=\sum_{\mathbf{k}}\left[G(\mathbf{k}) a_{\mathbf{k}}^{\dagger} \sigma_{g s}+\right.$ H.c. $]$, which reads

$$
G(\mathbf{k})=\frac{1}{N_{p}} \sum_{\alpha=1}^{N_{p}} g_{\mathbf{n}_{\alpha}} e^{-i \mathbf{k} \cdot \mathbf{n}_{\alpha}} .
$$

In this case $G_{1 \mathrm{D}}(\mathbf{k}) \propto 1+e^{-i\left(k_{x}+k_{y}\right)}$, which satisfies $G_{1 \mathrm{D}}\left(k_{x}, \pm \pi-k_{x}\right) \equiv 0$. Thus, the giant $\mathrm{QE}$ is effectively uncoupled from the $\mathbf{k}$ modes responsible of the forward or backward direction in the diagonal where the giant $\mathrm{QE}$ is coupled to, and does not decay into them. After having numerically seen how $H_{\text {int,eff }}$ emerges from $H_{\text {int,mov }}(t)$ for this example, from now on we use $H_{\text {int,eff }}$ to analyze the dynamics.

Trapped emission.- Let us now consider that the QE moves around four positions, i.e., $( \pm 1,0),(0, \pm 1)$. The effective $\mathbf{k}$ coupling will be $G_{\text {trap }}(\mathbf{k})=g\left(e^{i k_{x}}+e^{-i k_{x}}+\right.$ $\left.e^{i k_{y}}+e^{-i k_{y}}\right) / 4$, which cancels the coupling among the four resonant $\mathbf{k}$ lines. Thus, the giant $\mathrm{QE}$ will not decay, while keeping some of the photon population trapped between the four positions (not shown). As in the 1D counterpart [58], these confined photons will mediate coherent interactions between these decoherence-free QEs.

Filtering non-Markovian emission.-Another feature that can be achieved by coupling to few lattice sites is the effective decoupling from zero-group velocity terms occurring at $\mathbf{k}=(0, \pm \pi)$ and $( \pm \pi, 0)$. For that, we can couple the QE to the positions $( \pm 1, \pm 1),( \pm 1, \mp 1)$, with an alternating \pm 1 phase, such that $G_{\text {pur }}(\mathbf{k})=g \sin \left(k_{1}\right)$ $\sin \left(k_{2}\right)$. This has two consequences: first, the QE shows a more homogeneous directional emission, as observed in Fig. 3(a). Second, it smooths the effective spectral density probed by the QE, as plotted in Fig. 3(b), making its dynamics more Markovian. Thus, giant QEs provide a way of decoupling directional emission from non-Markovian dynamics in van Hove singularities.

Reverse design: Chiral and $V$-type emission.-In the previous examples it was possible to guess the spatial 


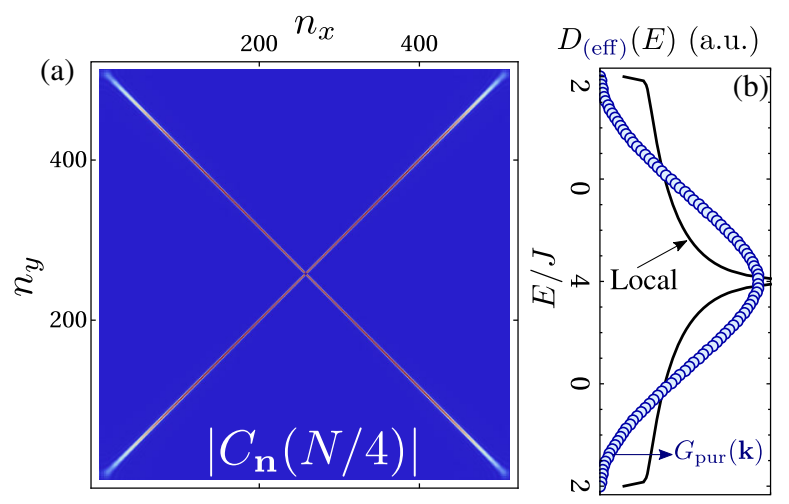

FIG. 3. (a) Bath probability amplitude at a time $t J=N / 4$ for a single giant QE coupled with $G_{\text {pur }}(\mathbf{k}), g=0.1 J$ and bath linear size is $N=512$. (b) Comparison of the density of states of the structured bath (black), with the effective spectral density which includes the $\mathbf{k}$ dependence of $G_{\text {pur }}(\mathbf{k})$, i.e., $D_{\text {eff }}(E)=$ $\sum_{\mathbf{k}}\left|G_{\text {pur }}(\mathbf{k})\right|^{2} \delta(E-\omega(\mathbf{k}))$.

couplings required to obtain the desired behavior. An alternative approach consists of first guessing the $G(\mathbf{k})$ required to obtain a given behavior, and then Fourier transforming it to get the spatial dependent couplings; that is

$$
G(\mathbf{n})=\frac{1}{N^{2}} \sum_{\mathbf{k}} G(\mathbf{k}) e^{-i \mathbf{k} \cdot \mathbf{n}}
$$

For example, let us imagine we want to obtain perfect chiral emission in one or two orthogonal directions out of the four appearing with local couplings. It is easy to see that

$$
\begin{array}{r}
G_{\mathrm{chi}}(\mathbf{k}) \propto \cos \left(\frac{k_{1}-k_{2}}{2}\right)\left[1+\sin \left(\frac{k_{1}+k_{2}}{2}\right)\right], \\
G_{\mathrm{V}}(\mathbf{k}) \propto\left[1-\sin \left(\frac{k_{1}-k_{2}}{2}\right)\right]\left[1-\sin \left(\frac{k_{1}+k_{2}}{2}\right)\right],
\end{array}
$$

cancels the coupling to the light emitted in three (or two) of the four directions, respectively. Then, using Eq. (7) we obtain the spatial profile of the couplings whose absolute value $|G(\mathbf{n})|$ is plotted in the inset of Figs. 4(a) and 4(b). The coupling spatial pattern is more intricate than in the previous situations because it requires adding complex phases (not shown), and involves the coupling to many lattice sites. Since this will be experimentally challenging, one needs to adopt a truncation strategy in which one approximates the sum by a finite number $n_{\text {tr }}$ of terms, $G(\mathbf{n}) \approx G_{\text {trunc }}\left(\mathbf{n} ; n_{\text {tr }}\right)$. This is what we do in Figs. 4(a) and $4(\mathrm{~b})$, where we observe that even for a small $n_{\text {tr }}$, the QE emits approximately with the desired behavior. Finally, in Figs. 4(c) and 4(d) we show how by increasing $n_{\text {tr }}$, the light collimated in the desired directions can go close to $100 \%$.

Interactions. - Let us finally point how these unconventional emission patterns will translate into exotic $\mathrm{QE}$

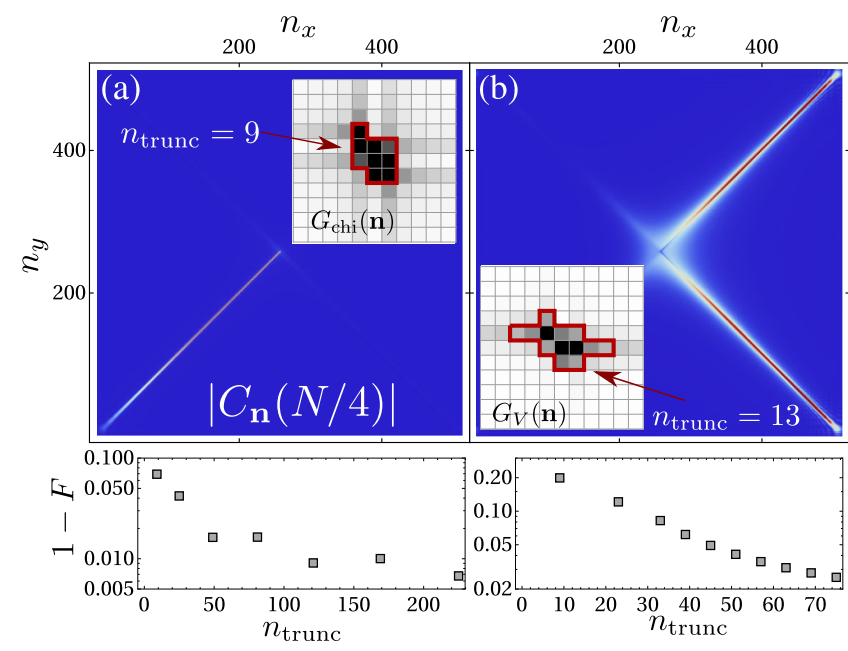

FIG. 4. (a),(b) Bath probability amplitude at a time $t J=N / 4$ for a single giant $\mathrm{QE}$ coupled with $G_{\text {trunc }}\left(\mathbf{n} ; n_{\mathrm{tr}}\right)$, respectively, $g=0.1 J$ and bath linear size is $N=512$. Inset: Corresponding spatial coupling profile $|G(\mathbf{n})|$ using Eq. (7). The red outline indicates the truncation we use to plot the figure. (c)-(d) $1-F$, where $F$ is the fraction of the emission into the desired directions for the parameters of panels (a) and (b), as a function of the number of terms in the sum $G_{\text {trunc }}\left(\mathbf{n} ; n_{\text {tr }}\right)$.

interactions when $N_{e}$ QEs are coupled to the bath. For simplicity, let us assume that each QE has a k-dependent coupling $G_{j}(\mathbf{k})=G(\mathbf{k}) e^{-i \mathbf{k} \cdot \mathbf{n}_{j}}$, where $e^{-i \mathbf{k} \cdot \mathbf{n}_{j}}$ is a global phase factor which indicates the giant $\mathrm{QE}$ central position $\left(\mathbf{n}_{j}\right)$, and $G(\mathbf{k})$ is a common $\mathbf{k}$-dependent coupling defined by the nonlocal couplings around the position $\mathbf{n}_{j}$. Then, if we trace out the bath degrees of freedom under the Born-Markov approximation, the QE reduced density matrix $(\rho)$ dynamics is governed by [72] $\partial_{t} \rho=i\left[\rho, H_{S}+\right.$ $\left.\sum_{i, j} J_{i j} \sigma_{e g}^{i} \sigma_{g e}^{j}\right]+\sum_{i, j}\left(\gamma_{i j} / 2\right)\left(2 \sigma_{g e}^{i} \rho \sigma_{e g}^{j}-\sigma_{e g}^{j} \sigma_{g e}^{i} \rho-\rho \sigma_{e g}^{j} \sigma_{g e}^{i}\right)$. The collective interactions $J_{i, j}, \gamma_{i j}$ are

$$
i \gamma_{i j}+J_{i j}=\frac{1}{N^{2}} \sum_{\mathbf{k}} \frac{|G(\mathbf{k})|^{2}}{\omega_{e}-\omega(\mathbf{k})-i 0^{+}} e^{i \mathbf{k} \cdot\left(\mathbf{n}_{i}-\mathbf{n}_{j}\right)}
$$

whose integrand is directly connected with the asymptotic emission pattern described in Eq. (5). This tells us, for example, that using the couplings $G_{1 \mathrm{D}}(\mathbf{k})$ or $G_{\text {chi }}(\mathbf{k})$ we will be able to simulate standard or chiral [73] waveguide QED couplings in two-dimensional baths, as well as other $\mathrm{QE}$ interactions with no counterpart in other setups, i.e., V-type collective decays.

Conclusions.-Summing up, we propose a method to engineer effective nonlocal light-matter couplings using ultracold atoms in dynamical state-dependent optical lattices. Controlling the confinement and relative position of two optical potentials, one can simulate giant atoms coupled to structured photonic baths in one, two, and three dimensions. Irrespective of the implementation, we also numerically illustrate the potential of giant emitters to yield 
unconventional quantum optical behavior when coupled to a two-dimensional structured bath. In particular, we exploit the interplay between the structured energy dispersion and nonlocal couplings to obtain exotic emission patterns and collective dissipative interactions. These recipes can be immediately adapted to other platforms where such nonlocal couplings can be engineered, or to higher dimensions [70,74].

Beyond the fundamental interest of the phenomena explored throughout the Letter, there are many possible follow-up applications. From the quantum simulation perspective, giant atoms provide a very flexible playground to probe equilibrium $[46,47]$ and nonequilibrium manybody physics $[75,76]$ with no analog in other setups. In addition, one can increase their tunability exploiting the interplay with the polarization degree of freedom [77-79], or through additional bath engineering [80]. Other possibilities, if one is able to engineer it with optical photons, is to exploit the multidirectional chiral emission to transfer simultaneously quantum information into several nodes, or for generating high-dimensional photonic entangled states [81], which can be used for fault-tolerant measurement based quantum computation [82].

J. I. C. acknowledges the ERC Advanced Grant QENOCOBA under the EU Horizon 2020 program (Grant Agreement No. 742102). C. S. M. is supported by the Marie Sklodowska-Curie Fellowship QUSON (Project No. 752180). A. G. T. acknowledges very useful discussions with J. Knörzer and the critical reading of the manuscript of T. Ramos. A. G. T. acknowledges support from CSIC Re-search Platform on Quantum Technologies PTI-001.

*a.gonzalez.tudela@csic.es

[1] E. M. Purcell, H. C. Torrey, and R. V. Pound, Phys. Rev. 69, 37 (1946).

[2] S. Haroche and D. Kleppner, Phys. Today 42, No. 1, 24 (1989).

[3] R. Miller, T. E. Northup, K. M. Birnbaum, A. Boca, A. D. Boozer, and H. J. Kimble, J. Phys. B 38, S551 (2005).

[4] H. J. Kimble, Nature (London) 453, 1023 (2008).

[5] E. Vetsch, D. Reitz, G. Sagué, R. Schmidt, S. T. Dawkins, and A. Rauschenbeutel, Phys. Rev. Lett. 104, 203603 (2010).

[6] J. D. Thompson, T. G. Tiecke, N. P. de Leon, J. Feist, A. V. Akimov, M. Gullans, A. S. Zibrov, V. Vuletic, and M. D. Lukin, Science 340, 1202 (2013).

[7] A. Goban, C.-L. Hung, S.-P. Yu, J. Hood, J. Muniz, J. Lee, M. Martin, A. McClung, K. Choi, D. Chang, O. Painter, and H. Kimblemblrm, Nat. Commun. 5, 3808 (2014).

[8] J.-B. Béguin, E. M. Bookjans, S. L. Christensen, H. L. Sørensen, J. H. Müller, E. S. Polzik, and J. Appel, Phys. Rev. Lett. 113, 263603 (2014).

[9] P. Lodahl, S. Mahmoodian, and S. Stobbe, Rev. Mod. Phys. 87, 347 (2015).
[10] A. Sipahigil, R. E. Evans, D. D. Sukachev, M. J. Burek, J. Borregaard, M. K. Bhaskar, C. T. Nguyen, J. L. Pacheco, H. A. Atikian, C. Meuwly, R. M. Camacho, F. Jelezko, E. Bielejec, H. Park, M. Lončar, and M. D. Lukin, Science 354, 847 (2016).

[11] N. V. Corzo, B. Gouraud, A. Chandra, A. Goban, A. S. Sheremet, D. V. Kupriyanov, and J. Laurat, Phys. Rev. Lett. 117, 133603 (2016).

[12] H. L. Sørensen, J.-B. Béguin, K. W. Kluge, I. Iakoupov, A. S. Sørensen, J. H. Müller, E. S. Polzik, and J. Appel, Phys. Rev. Lett. 117, 133604 (2016).

[13] P. Solano, P. Barberis-Blostein, F. K. Fatemi, L. A. Orozco, and S. L. Rolston, Nat. Commun. 8, 1857 (2017).

[14] D. E. Chang, J.S. Douglas, A. González-Tudela, C.-L. Hung, and H. J. Kimble, Rev. Mod. Phys. 90, 031002 (2018).

[15] Y. Liu and A. A. Houck, Nat. Phys. 13, 48 (2017).

[16] N. M. Sundaresan, R. Lundgren, G. Zhu, A. V. Gorshkov, and A. A. Houck, Phys. Rev. X 9, 011021 (2019).

[17] M. Mirhosseini, E. Kim, V. S. Ferreira, M. Kalaee, A. Sipahigil, A. J. Keller, and O. Painter, Nat. commun. 9, 3706 (2018).

[18] I. de Vega, D. Porras, and J. Ignacio Cirac, Phys. Rev. Lett. 101, 260404 (2008).

[19] C. Navarrete-Benlloch, I. de Vega, D. Porras, and J. I. Cirac, New J. Phys. 13, 023024 (2011).

[20] T. Ramos, B. Vermersch, P. Hauke, H. Pichler, and P. Zoller, Phys. Rev. A 93, 062104 (2016).

[21] B. Vermersch, T. Ramos, P. Hauke, and P. Zoller, Phys. Rev. A 93, 063830 (2016).

[22] L. Krinner, M. Stewart, A. Pazmino, J. Kwon, and D. Schneble, Nature (London) 559, 589 (2018).

[23] S. John and T. Quang, Phys. Rev. A 50, 1764 (1994).

[24] Q.-J. Tong, J.-H. An, H.-G. Luo, and C. H. Oh, Phys. Rev. A 81, 052330 (2010).

[25] P. Longo, P. Schmitteckert, and K. Busch, Phys. Rev. Lett. 104, 023602 (2010).

[26] S. Garmon, T. Petrosky, L. Simine, and D. Segal, Fortschr. Phys. 61, 261 (2013).

[27] E. S. Redchenko and V. I. Yudson, Phys. Rev. A 90, 063829 (2014).

[28] F. Lombardo, F. Ciccarello, and G. M. Palma, Phys. Rev. A 89, 053826 (2014).

[29] E. Sánchez-Burillo, D. Zueco, L. Martín-Moreno, and J. J. García-Ripoll, Phys. Rev. A 96, 023831 (2017).

[30] V. P. Bykov, Sov. J. Quantum Electron. 4, 861 (1975).

[31] S. John and J. Wang, Phys. Rev. Lett. 64, 2418 (1990).

[32] G. Kurizki, Phys. Rev. A 42, 2915 (1990).

[33] S. Tanaka, S. Garmon, and T. Petrosky, Phys. Rev. B 73, 115340 (2006).

[34] G. Calajó, F. Ciccarello, D. Chang, and P. Rabl, Phys. Rev. A 93, 033833 (2016).

[35] T. Shi, Y.-H. Wu, A. González-Tudela, and J. I. Cirac, Phys. Rev. X 6, 021027 (2016).

[36] P. Facchi, M. S. Kim, S. Pascazio, F. V. Pepe, D. Pomarico, and T. Tufarelli, Phys. Rev. A 94, 043839 (2016).

[37] F. Galve, A. Mandarino, M. G. Paris, C. Benedetti, and R. Zambrini, Sci. Rep. 7, 42050 (2017).

[38] A. González-Tudela and J. I. Cirac, Phys. Rev. Lett. 119, 143602 (2017). 
[39] A. González-Tudela and J. I. Cirac, Phys. Rev. A 96, 043811 (2017).

[40] F. Galve and R. Zambrini, Ann. Phys. (Berlin) 530, 1800017 (2018).

[41] A. Asenjo-Garcia, M. Moreno-Cardoner, A. Albrecht, H. J. Kimble, and D. E. Chang, Phys. Rev. X 7, 031024 (2017).

[42] E. Shahmoon, D. S. Wild, M. D. Lukin, and S. F. Yelin, Phys. Rev. Lett. 118, 113601 (2017).

[43] A. W. Glaetzle, K. Ender, D. S. Wild, S. Choi, H. Pichler, M. D. Lukin, and P. Zoller, Phys. Rev. X 7, 031049 (2017).

[44] J. Perczel, J. Borregaard, D. E. Chang, H. Pichler, S. F. Yelin, P. Zoller, and M. D. Lukin, Phys. Rev. Lett. 119, 023603 (2017).

[45] A. Albrecht, L. Henriet, A. Asenjo-Garcia, P. B. Dieterle, O. Painter, and D. E. Chang, New J. Phys. 21, 025003 (2019).

[46] J. S. Douglas, H. Habibian, C.-L. Hung, A. Gorshkov, H. J. Kimble, and D. E. Chang, Nat. Photonics 9, 326 (2015).

[47] A. González-Tudela, C.-L. Hung, D. E. Chang, J. I. Cirac, and H. Kimble, Nat. Photonics 9, 320 (2015).

[48] E. Shahmoon, P. Grišins, H. P. Stimming, I. Mazets, and G. Kurizki, Optica 3, 725 (2016).

[49] A. González-Tudela and J. I. Cirac, Phys. Rev. A 97, 043831 (2018).

[50] M. V. Gustafsson, T. Aref, A. F. Kockum, M. K. Ekström, G. Johansson, and P. Delsing, Science 346, 207 (2014).

[51] R. Manenti, A. F. Kockum, A. Patterson, T. Behrle, J. Rahamim, G. Tancredi, F. Nori, and P. J. Leek, Nat. Commun. 8, 975 (2017).

[52] A. Noguchi, R. Yamazaki, Y. Tabuchi, and Y. Nakamura, Phys. Rev. Lett. 119, 180505 (2017).

[53] A. N. Bolgar, J. I. Zotova, D. D. Kirichenko, I. S. Besedin, A. V. Semenov, R. S. Shaikhaidarov, and O. V. Astafiev, Phys. Rev. Lett. 120, 223603 (2018).

[54] B. A. Moores, L. R. Sletten, J. J. Viennot, and K. W. Lehnert, Phys. Rev. Lett. 120, 227701 (2018).

[55] A. Ciani and D. P. DiVincenzo, Phys. Rev. B 96, 214511 (2017).

[56] A. Frisk Kockum, P. Delsing, and G. Johansson, Phys. Rev. A 90, 013837 (2014).

[57] L. Guo, A. Grimsmo, A. F. Kockum, M. Pletyukhov, and G. Johansson, Phys. Rev. A 95, 053821 (2017).

[58] A. F. Kockum, G. Johansson, and F. Nori, Phys. Rev. Lett. 120, 140404 (2018).

[59] D. Jaksch, H.-J. Briegel, J. I. Cirac, C. W. Gardiner, and P. Zoller, Phys. Rev. Lett. 82, 1975 (1999).

[60] A. Sørensen and K. Mølmer, Phys. Rev. Lett. 83, 2274 (1999).

[61] E. Jané, G. Vidal, W. Dür, P. Zoller, and J. Cirac, Quantum Inf. Comput. 3, 15 (2003).
[62] I. Bloch, J. Dalibard, and W. Zwerger, Rev. Mod. Phys. 80, 885 (2008).

[63] A. Rubio-Abadal, J.-y. Choi, J. Zeiher, S. Hollerith, J. Rui, I. Bloch, and C. Gross, arXiv:1805.00056.

[64] A. J. Daley, M. M. Boyd, J. Ye, and P. Zoller, Phys. Rev. Lett. 101, 170504 (2008).

[65] S. Snigirev, A. J. Park, A. Heinz, S. Wissenberg, J. Dalibard, I. Bloch, and S. Blatt, in Quantum Information and Measurement (Optical Society of America, 2017), pp. QT4A-2.

[66] L. Riegger, N. Darkwah Oppong, M. Höfer, D. R. Fernandes, I. Bloch, and S. Fölling, Phys. Rev. Lett. 120, 143601 (2018).

[67] When the $b$ optical potential is weaker, its atomic wave function delocalizes allowing for a nonlocal coupling, which, however, does not help to obtain the desired behavior.

[68] In practice the transition from one position to the other will be a smooth function, which should satisfy the adiabaticity condition at any time.

[69] N. Goldman and J. Dalibard, Phys. Rev. X 4, 031027 (2014).

[70] See Supplemental Material at http://link.aps.org/ supplemental/10.1103/PhysRevLett.122.203603 for more details on the derivation of the effective Hamiltonian in the high-frequency limit, how the figures were calculated, and considerations about the experimental setups.

[71] L. Van Hove, Phys. Rev. 89, 1189 (1953).

[72] G. W. Gardiner and P. Zoller, Quantum Noise, 2nd ed. (Springer-Verlag, Berlin, 2000).

[73] P. Lodahl, S. Mahmoodian, S. Stobbe, A. Rauschenbeutel, P. Schneeweiss, J. Volz, H. Pichler, and P. Zoller, Nature (London) 541, 473 (2017).

[74] A. González-Tudela and J. I. Cirac, Quantum 2, 97 (2018).

[75] T. Ramos, H. Pichler, A. J. Daley, and P. Zoller, Phys. Rev. Lett. 113, 237203 (2014).

[76] H. Pichler, T. Ramos, A. J. Daley, and P. Zoller, Phys. Rev. A 91, 042116 (2015).

[77] J. Perczel and M. D. Lukin, arXiv:1810.12815.

[78] J. Perczel, J. Borregaard, D. E. Chang, S. F. Yelin, and M. D. Lukin, arXiv:1810.12299.

[79] S.-P. Yu, J. A. Muniz, C.-L. Hung, and H. J. Kimble, arXiv:1812.08936.

[80] A. González-Tudela and F. Galve, ACS Photonics 6, 221 (2019).

[81] H. Pichler, S. Choi, P. Zoller, and M. D. Lukin, Proc. Natl. Acad. Sci. U.S.A. 114, 11362 (2017).

[82] H. J. Briegel, D. E. Browne, W. Dür, R. Raussendorf, and M. Van den Nest, Nat. Phys. 5, 19 (2009). 